\title{
Kindermord und verheimlichte Schwangerschaft in Hamburg im 18. Jahrhundert
}

Versuch einer soziologischen und sozialmedizinischen Analyse

Von Heinrich Rodegra, Mary Lindeman und Martin Ewald

\section{Einleitung}

Im 18. Jahrhundert war eine Zunahme der Delikte von Kindesaussetzung und Kindermord festzustellen, wie aus zahlreichen zeitgenössischen Presseberichten zu entnehmen ist. Eine eigentliche Kriminalstatistik im heutigen Sinn gab es noch nicht. Vereinzelt - hauptsächlich in Preußen - gibt es tabellarische Angaben, aus denen sich ein ungefähres Bild der Kriminalität jener Zeit gewinnen läßt; jedoch ist man beim Versuch, Berichte über die Zunahme jener Straftaten zu bestätigen, auf Archivalien angewiesen.

Die Zahl der Verhandlungen oder der protokollierten Verurteilungen gibt keinen direkten Aufschluß über die eigentliche Anzahl der Kindermorde, da gerade bei dieser Straftat eine große Dunkelziffer angesetzt werden muß, welche u. a. auch dadurch zu erklären ist, daß die Existenz des Opfers oft nur der Mutter oder einem kleinen Personenkreis bekannt war, der von der Schwangerschaft gewußt hat. «Normalerweise wird jedoch das Opfer eines Tötungsdeliktes meistens von mehreren Personen des bisherigen Lebenskreises vermißt; sein Verschwinden löst Nachforschungen aus, das Auffinden der Leiche führt zur Identifikation, das Vorleben enthält Hinweise auf den Täter. ${ }^{1}$

In engem Zusammenhang mit dem Kindermord stand das Delikt der «verheimlichten Schwangerschaft», also das Nichtmelden einer unehelichen Schwangerschaft bei der zuständigen Behörde, das ebenfalls strafrechtlich verfolgt und oft als «Absichtsdelikt» für eine Kindestötung angesehen wurde.

In dieser Arbeit soll versucht werden, anhand der vorliegenden Archivalien das Geschehen «Kindermord» und «Verheimlichte Schwangerschaft» für die Stadt Hamburg im 18. Jahrhundert aufzuzeigen und eine Statistik aufgrund der wenigen vorhandenen tabellarischen Angaben und Senatsprotokolle ${ }^{2}$ zu erstellen, die strafrechtlichen Konsequenzen zu erläutern, die soziologischen Hintergründe dieses Deliktes zu erhellen und die sozialmedizinischen Maßnahmen zu beleuchten, mit denen man versuchte, die Aufklärungsquote zu heben und durch Vorbeugung auf dem medizinischen und medizinalpolizeilichen Sektor sowie durch Verbesserung des Hebammenwesens dieses Problem anzugehen. Über diese Maßnahmen hinaus sollten Verschärfungen des strafrechtlichen Vorgehens gegen den 
Täter, aber auch gegen den Personenkreis, der im engen Kontakt mit einer ledigen Schwangeren stand, für eine Eindämmung dieses Verbrechens sorgen.

Als Quellen für das zahlenmäßige Vorkommen dieser Delikte in Hamburg, ihre sozialmedizinischen Folgen und die medizinalgesetzlichen Maßnahmen dienen Senatsprotokolle sowie die verschiedenen Apotheken- und Medizinalordnungen jener Zeit. Zeitgenössische Literatur und Presseberichte geben den Hintergrund für die Situationsschilderung, während die strafrechtliche Seite das Hamburger Stadtrecht von 1603, seine Ergänzungen und Mandate sowie ebenfalls Senatsprotokolle erläutern.

\section{Der Kindermord in Hamburg im 18. Jahrhundert}

Wie in anderen Teilen des deutschen Landes, so gibt auch für Hamburg die von den Behörden registrierte Zahl ausgesetzter, tot gefundener Neugeborener und überführter Fälle von Kindermord nur Anhaltspunkte über dieses Delikt; denn auch hier war die Dunkelziffer sehr hoch. Zwar richtete man sich immer wieder mit Hinweisen an die Bevölkerung und wurde nicht müde, in Ermahnungen auf die Pflicht hinzuweisen, bei Bekanntwerden von verheimlichten unehelichen Schwangerschaften unmittelbar dem Prätor ${ }^{3}$ Meldung zu erstatten.

Insbesondere bestand eine verschärfte Meldepflicht für die Hebammen, auf die im vierten Kapitel noch näher eingegangen wird. Neben dem Versuch einer genauen Registrierung dieser Delikte war jedoch das Hauptanliegen der Mandate, den Kindermord zu verhindern und schon die «Erzeugung unehelicher Kinder», die dem Staat in Zukunft eine Last sein könnten, zu «kontrollieren». Deshalb bestimmte das Mandat vom 3. Januar $1749^{4}$ :

«Es hat E. Hochedl. Rath beliebet, daß mit dem Anfange ieden Jahres die Anzahl der in dem vorigen Jahre in ieder Stadt-Gemeine getauften sowol ehelichen als unehelichen Kinder, nicht weniger der copulirten Personen, Communicanten und Verstorbenen, respective von den Hochw. Herren Patronen und Wohlw. Kirchspiel-Herren produciret werden möge.»

Ab 1742 sind, wenn auch sehr lückenhaft, in den Senatsprotokollen der Hansestadt Hamburg - die Protokolle der Zeit davor sind verbrannt - Aussagen über die Häufigkeit der «todtgefundenen Kinder» und der nachgewiesenen Kindermorde vorhanden, wobei jedoch für den ersteren Fall nicht festzustellen ist, ob es sich dabei jeweils um einen Mord oder um ein totgeborenes Kind ohne äußere Gewalteinwirkung handelte.

In der nachfolgenden Tabelle werden für einige Zeiträume des 18. Jahrhunderts die Zahl der tot gefundenen Neugeborenen, der nachgewiesenen und überführten Kindermorde sowie der verheimlichten Schwangerschaften ausgewiesen ${ }^{5}$. 
Tabelle 1: Tot gefundene Neugeborene, nachgewiesene und überführte Kindermorde sowie verheimlichte Schwangerschaften in Hamburg für einige Zeiträume des 18. Jahrhunderts.

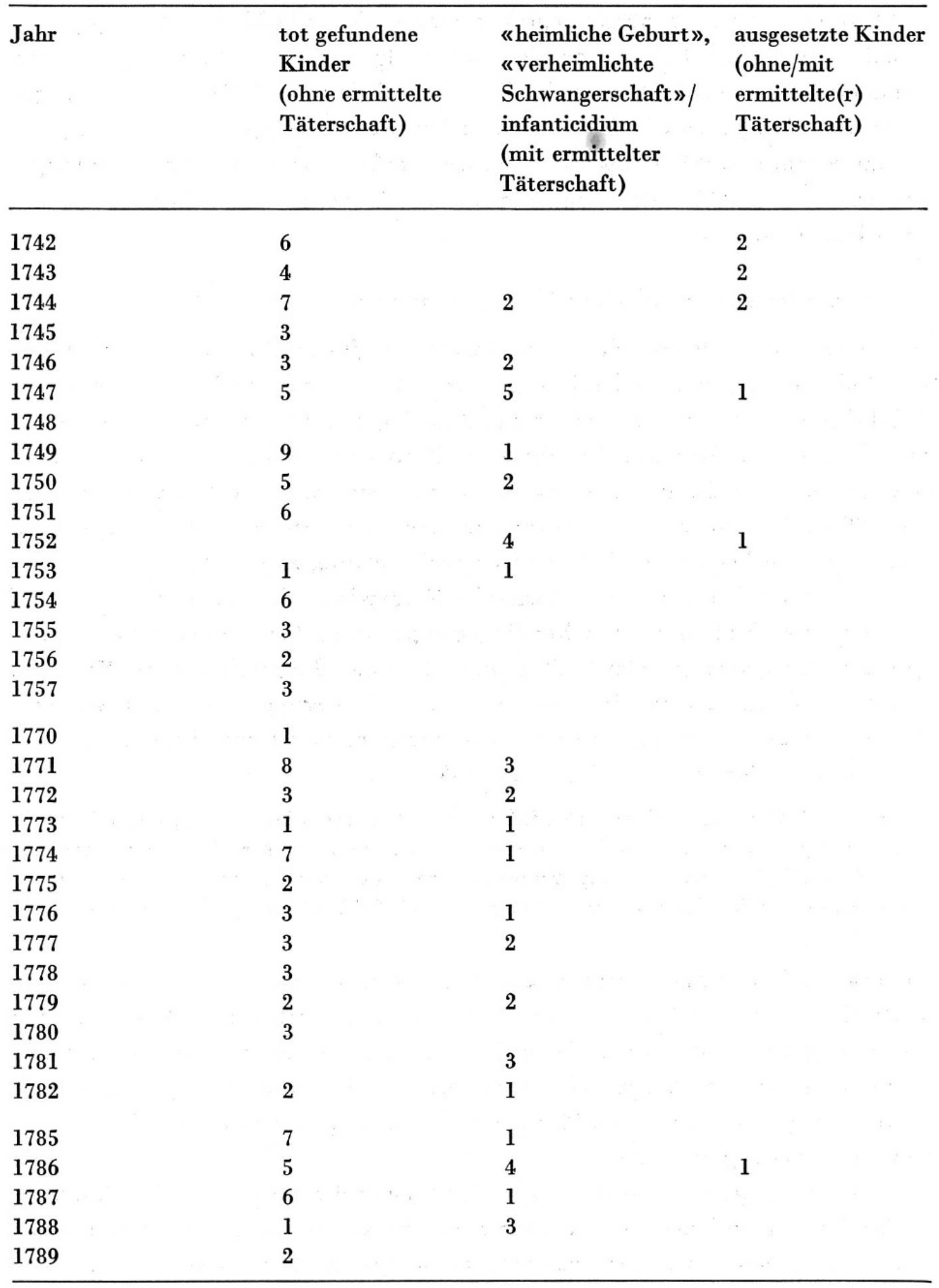




\begin{tabular}{llll}
\hline Jahr & $\begin{array}{l}\text { tot gefundene } \\
\text { Kinder } \\
\text { (ohne ermittelte } \\
\text { Täterschaft) }\end{array}$ & $\begin{array}{l}\text { «heimliche Geburt», } \\
\text { «verheimlichte } \\
\text { Schwangerschaft»/ } \\
\text { infanticidium } \\
\text { (mit ermittelter } \\
\text { Täterschaft) }\end{array}$ & $\begin{array}{l}\text { ausgesetzte Kinder } \\
\text { (ohne/mit } \\
\text { ermittelte(r) } \\
\text { Täterschaft) }\end{array}$ \\
\hline 1790 & & & \\
1791 & & 2 & \\
1792 & 1 & & \\
1793 & 3 & 3 & 2 \\
1794 & 7 & 1 & 1 \\
1795 & 1 & 1 & \\
1796 & & 1 & \\
1797 & & 2 & \\
1798 & 2 & 3 & \\
1799 & 1 & 1 & \\
1800 & 3 & & \\
\hline
\end{tabular}

Die nächste Tabelle bietet eine Übersicht der ehelich und unehelich getauften Kinder der Stadt Hamburg für einige Zeiträume des 18. Jahrhunderts ${ }^{6}$.

Tabelle 2: Statistik unehelicher Geburten: Stadtgebiet und Patronate (Vorstädte) St. Georg, Hamburger Berg (später St.Pauli), nicht Landgebiet. Teils nur ev.-luth. Getaufte, ohne «fremde Religionsverwandte».

\begin{tabular}{lllllll}
\hline Jahr & Getaufte & davon ehelich & unehelich & \multicolumn{2}{l}{ Einwohner } & \\
\hline 1712 & 3018 & 2971 & 47 & & ca. 75000 & $(1710)$ \\
1713 & 2758 & 2700 & 58 & & & \\
1714 & 2726 & 2663 & 63 & & \\
1715 & 3012 & 2970 & 42 & & & \\
& & & & & & \\
1768 & 2817 & 2572 & 245 & & & \\
1770 & 2764 & 2538 & 226 & & & \\
1775 & 2606 & 2343 & 263 & & & \\
1780 & 2680 & 2425 & 255 & & & \\
1785 & 3003 & 2691 & 312 & & & \\
1790 & 2792 & 2480 & 312 & & & \\
1793 & 2880 & 2557 & 323 & & & \\
1795 & 2830 & 2517 & 313 & & & \\
1797 & 3355 & 2953 & 402 & & & \\
1799 & 3595 & 3158 & 437 & & & \\
1800 & 4019 & 3521 & 498 & & & \\
\hline
\end{tabular}


Bevorzugte Stellen einer Aussetzung für die «todtgefundenen Kinder» waren die Kirchhöfe; auch wurde als Fundort häufig «im Wasser» bei den verschiedenen Fleeten ${ }^{7}$ angegeben. Bei den in der Zeit von 1771-1803 registrierten Kindermorden findet sich nur in drei Fällen bei den Angaben über die Mutter die Bezeichnung "verheiratet»; stets handelte es sich hier jedoch um Kinder, die in «ehebrecherischen Verhältnissen» gezeugt worden waren.

\section{Strafrechtlicher Tatbestand und soziologische Hintergründe der verheimlichten Schwangerschaft und der Kindermorde}

\section{a) Der strafrechtliche Tatbestand}

Schon im 16. Jahrhundert wurden gesetzliche Vorschriften erlassen, Schwangerschaften bei unverheirateten oder verwitweten Frauen zu melden. Diese Vorschriften hatten bis zum 18. Jahrhundert noch Gültigkeit. Nach einem Gesetz von Heinrich II. von Frankreich (1556) war z. B. jede schwangere unverheiratete Frau oder Witwe angewiesen, ihre Schwangerschaft dem Magistrat oder einer Hebamme - dieser oblag wiederum die Pflicht der Weiterleitung der Meldung an die entsprechende Behörde - anzuzeigen. Der Magistrat war dann gehalten, die Zeitdauer der Schwangerschaft, den Schwängerer und den Ort der Schwängerung zu ermitteln. Besonders den Hebammen wurde nahegelegt, diese Fragen «unter der Geburt» zu stellen, da die Frau dann eher bereit wäre, «die wahre Quelle ihrer Schmerzen [den Vater des Kindes] zu nennen ". ${ }^{8}$

Die Verheimlichung einer Schwangerschaft oder, wie es in den Mandaten ausgedrückt ist, « eine Schwangerschaft verhehlen oder verheimlichen » oder «heimlich ohne weibliche Hülfen geboren haben», galt schon als Beweis, daß im voraus geplant war, das Kind «an der Seiten zu nehmen», das Neugeborene zu ermorden oder "wegzulegen». Die Meldepflicht für eine Schwangerschaft bestand in Hamburg offensichtlich nur für die unverheiratete Schwangere und nicht für die verheiratete Frau.

Der Begriff Kindermord (Kindestötung, Kindesmord, lat. infanticidium) tritt uns in den Quellen bis tief ins 19. Jahrhundert in zweierlei Bedeutung entgegen: Im weiteren Sinn wird unter Kindermord die vorsätzliche Tötung eines Kindes durch die Eltern, insbesondere die Mutter, verstanden; es handelt sich also um einen Spezialfall des Verwandtenmordes. In einer engeren speziellen Anwendung bezeichnet Kindermord die vorsätzliche Tötung eines neugeborenen, außerehelich gezeugten Kindes während oder gleich nach der Geburt; Täterin kann nur die Mutter sein. Dieser Tatbestand des Kindermordes im eigentlichen Sinne 
wurde von der gemeinrechtlichen Strafrechtslehre am Ende des 18. Jahrhunderts entwickelt und verfeinert ${ }^{9}$.

Die strafrechtlichen Kriterien für Kindermord für das 18. Jahrhundert finden sich in der «Carolina», der «Peinlichen Halsgerichtsordnung» (P.H.G.O.) von 1532, im Artikel 130 (Straff der weiber so jre Kinder tödten); die verheimlichte Schwangerschaft wird in den Artikeln 35 und 36 behandelt (Von heymlichem Kinderhaben, und tödten durch jre Mütter genugsam Anzeygung).

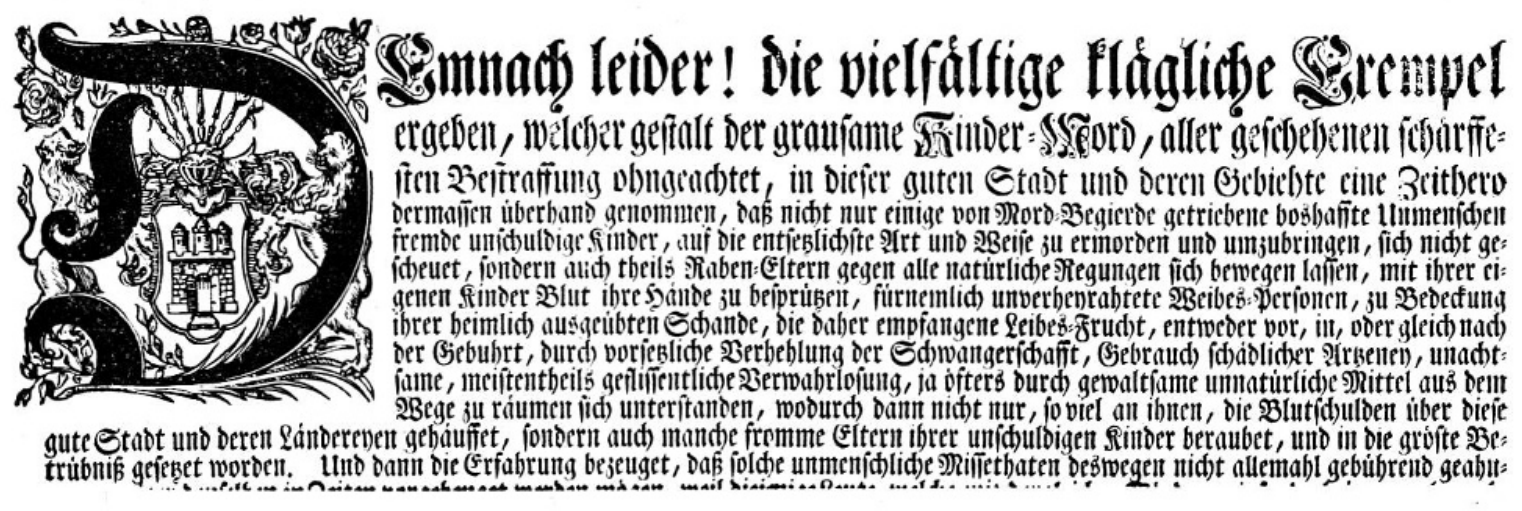

Abb. 1. Mandat gegen Kindermord vom 18.10.1724, Ausschnitt (Staatsarchiv Hamburg: Senat, Cl. VII Lit. $\mathrm{M}^{\mathrm{e}} \mathrm{Nr} .8$ Vol. $^{\mathrm{b}}$ )

In Hamburg finden wir in einem Mandat vom 18. Oktober 1724 (Abb.1) die Androhung von Bestrafung für «heimliche Schwangerschafften», auch ausgedehnt auf das «Mitwissen». Veranlaßt war dies durch die Zunahme der Delikte in jener Zeit. In diesem Mandat heißt es ${ }^{10}$ :

«So hat E. E. Raht so-wohl zu Aufrechterhaltung der Justiz, als auch zur Befreyung seines Gewissens sich gemüßiget befunden, wie zufoderst alle und jede dieser Stadt Bürger, Einwohner und Unterthanen, von vorgedachter irrigen Meinung und harten Begegnung abzumahnen, und ihnen das Gewissen dahin zu schärffen, daß sie allerdings in der grösten Seelen-Gefahr sich befinden, wenn sie dergleichen heimliche Schwangerschafften, oder die von ein und anderem vorhabende Mordthaten und desfalls geschöpfte Vermuthungen nicht zeitig entdecken, so auch denenselben, und Insonderheit denen Heb-Ammen oder so genannten Bade-Müttern, ernstlich zu befehlen und anzudeuten, daß, daferne sie ins künftige so wohl von heimlichen Schwangerschafften, als vorhabenden oder auch schon würcklich vollbrachten Kinder-Mordten etwas in Erfahrung bringen, und solches nicht ohngesäumt entdecken, und den Herren Prätoribus anzeigen werden, sie als Gewissenlose Mitschuldige angesehen, und mit hoher willkührlicher, auch dem Befinden nach mit Gefängniß und anderer harten Leibes-Strafe ohnausbleiblich beleget werden sollen.»

Dieses Mandat wurde in Anlehnung an das Hamburgische Stadtrecht von 1603, Part. IV, Artikel 23, erlassen. Dieser Artikel enthielt analog dem Artikel 137 der P.H.G.O. folgende Strafandrohung: 
«Strafe derjenigen, die ihre Eltern, Kinder, Schwestern, Brüder, oder nahe verwandte Freunde ermorden.

Wofern die Kinder ihre Eltern, oder die Eltern ihre Kinder, aus bösem teuflischem Vorsatz mördlich umbringen, oder sonst durch Gift ihres Lebens berauben, so soll solcher Eltern- oder Kindermörder mit glüenden Zangen angegriffen und darauf lebendig mit dem Rade getödtet werden. Da auch solcher schrecklicher Mord an Schwester, oder Gebrüdern, oder andern nahe verwandten Bluts-Freunden würde begangen, auf den Fall soll solcher Mörder, mit dem Rade am Leben gestrafet werden. Imgleichen sollen auch diejenigen, die ihre eigene Kinder umbringen, oder aus teuflischem Gemüthe, die lebendige Frucht ihres Leibes abtreiben, und also des Lebens berauben, an ihrem Leben wieder gestrafet, und mit dem Schwerdt hingerichtet, oder im Wasser erträncket, oder lebendig begraben werden.»

Diese Gesetze mußten von der P.H.G.O. übernommen werden, da das Hamburgische Recht keine besonderen Artikel vom "parricidium», dem «Verwandten- oder Nächstenmord», enthielt. Die P.H.G.O. stellte die Strafe bei einem Eltern- oder Kindermord dem Brudermord hinsichtlich des Strafmaßes gleich und forderte die «Strafe des Rades». Jedoch wurde häufig diese Strafe in «die Strafe des Schwerdts» gemildert. Weitere Hinrichtungsmaßnahmen waren im Zuge dieser «Milderung» das «Ertränken» oder «Lebendig Begraben».

In Hamburg wurde, wie aus den Senatsprotokollen und den StadtrechtsKommentaren zu ersehen ist, zwar auch die Todesstrafe verhängt; in vielen Fällen jedoch erfolgte eine Umwandlung in Zuchthaus- oder Gefängnisstrafe mit anschließender Stadtverweisung, wobei die Jugend der Angeklagten, ihr bisheriger Lebenswandel, negative Beeinflussung durch den Kindesvater als mildernde Umstände geltend gemacht wurden. Weitere Kriterien für das Strafmaß waren auch die Herkunft der Beklagten - ob sie aus Hamburg gebürtig oder aus der Fremde gekommen waren -, das Elternhaus, ihr Alter oder die «geistige und körperliche Gesundheit».

Häufig findet man zusätzlich zur Gefängnis- oder Zuchthausstrafe «Aufstellung am Pranger», womit man einen abschreckenden Effekt erzielen wollte. So heißt es in einem Gerichtsurteil vom 4.April $1727^{11}$,

«daß Gefangene Inquisitin und peinlich Angeklagte mit der von Fiscali gebetenen Todes-Strafe zwar zu verschonen, iedennoch, wegen dessen, daß sie sowohl ihre Schwangerschaft vorsetzlich verhehlet, als auch die vor, in und nach der Geburt augenblicklich zu habende Hülfe muthwillig verabsäumet, ihr zur wohlverdienten Strafe, andern dergleichen unzüchtigen und ihre Schwangerschaft verhehlenden Weibesbildern aber zum abschreckenden Exempel, am Pranger zu stellen, mit Ruthen zu streichen, und auf 20 Jahre in hiesiges Spinnhaus zu setzen sey» (siehe auch Abb. 2 und 3).

Das Spinnhaus wurde im Jahre 1669 gleich «ad perpetuos carceres» erbaut. Dieses Haus war das eigentliche Gefängnis und nahm anfangs - nach der Haus- 


\section{Shieftge Zorfálle. Urtbeil und; volljogene Btrafe, ben 15 Dec. 1792.}

Fifcalis contr. Doroth. 经lifab. Odwat. aen. Ertannt eill Şodprciß̄l. Dbergeridht, ons bie Pieberidtl. Fimung yom 23 Nov. a. c. zu conform, \& ju reform. folgenberges Rallt uno alfo, bas gefangene Inquifitin petnl. angeflagt, Da Diefelbe fith Towobl mabrens iber moblbewuften Ectroangers fart, surch frôfliche Rerbeimlidung ber. felbin, alfo aud ben und nad ber Beburt, felb|t ourd firåf. Derfaumung fremoer Sủlfe uno unnatưrlichen Bernad)!ảsigungen ibree getragenea leibesbuirde von beren bamaligen Soot, fie felbit teineśnegés gemißg gemejen, fich sine ảuferft ftrafbare Bernactlä̉igung fouldig gemadst, Der in ben Acten vorfom. menden bifonbern ghilbrungegrilloe wegen, gronar mit ber auf oergl. Berbridien gefigtell or. bentlichen Etrafe ju oerfabolien, jeood ibr jur moblocrdienten extrafe, andern orglitaen Iectifinnigin $\mathfrak{P}$ irfonen aber zum abfaria, orn Bevfptels, sine Esuricelang mit Rutben umben Salk, an ben 9ranger zu fellen, uno onll nád alt auf 10 Jabre til cas biefige Epinn. baus ju figin, nach beren yblaufe aber, aus diefic Etadt und beren Beblete nuf Zeitiebent au verfiften. D. R. W.

$A b b$. 2. Aus: Wöchentliche gemeinnützige Nachrichten von und für Hamburg vom 19.12.1792, Spalte 705 (Staatsarchiv Hamburg) 


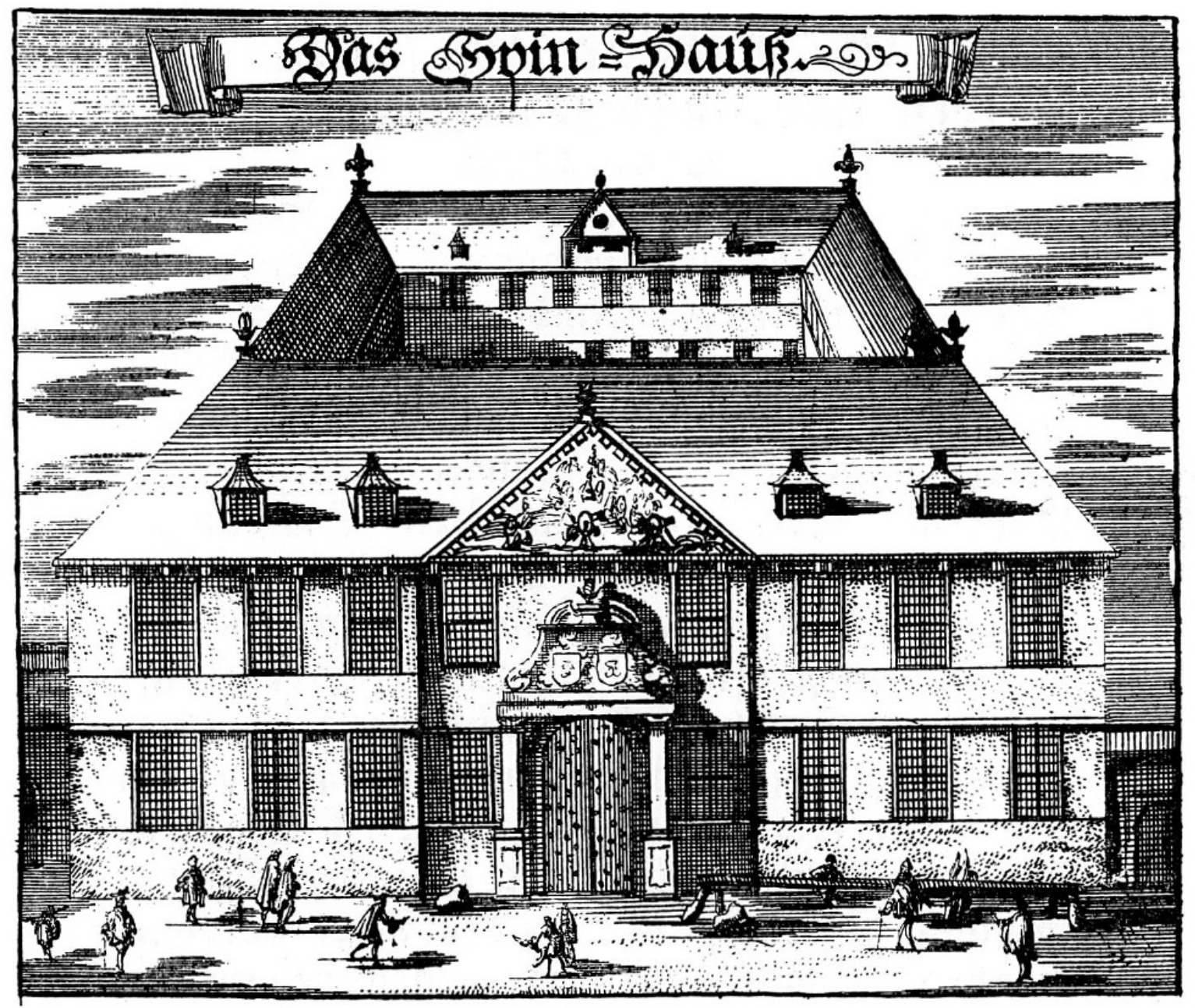

Abb. 3. Das Spinnhaus um 1690 (Staatsarchiv Hamburg: Pl 131-6/225/21)

ordnung vom 12. Mai 1669 - besonders «Huren und Diebe» auf, danach aber auch Schwerverbrecher. Die Verwahrung im Spinnhaus war die Folge einer erkannten und vollzogenen kriminellen Bestrafung und erfolgte nach einem gerichtlichen Prozeß. Außerdem hatte das Spinnhaus noch die Funktion einer «polizeilichen Entbindungsanstalt». Unverheiratete Schwangere konnten beim Prätor um ein «freies Wochenbett» bitten und wurden von ihm an den Provisor des Spinnhauses verwiesen, der sie zur «Haltung des Wochenbettes» annehmen mußte. Die Schwangere wurde von der Ratswehmutter entbunden. Es bestand eine Verfügung, «daß keine von den liederlichen Weibsbildern, welche geschickt worden waren, ohne der Herren Prätorum Vorwissen vom Spinnhause dimittiret werden "; sie hatten dort noch etwa für ein halbes Jahr zu arbeiten, um auf diesem Wege einen Teil der Entbindungskosten zurückzuerstatten ${ }^{12}$. Im Jahre 1796 wurde zusätzlich in diesem Areal noch eine Entbindungsanstalt als Einrichtung 
der Allgemeinen Armenanstalt errichtet. In diesem Entbindungshaus wurden jährlich ca. 100 Entbindungen vorgenommen. Der Bau dieser Anstalt war erforderlich geworden, um die vielen Schwangeren aus den unteren sozialen Schichten aufnehmen zu können, die aus Hamburg selbst stammten oder aus den umliegenden Landgemeinden nach Hamburg strömten, um hier Hilfe zu suchen ${ }^{13}$.

\section{b) Soziologische Hintergründe}

Das 17. und 18. Jahrhundert hatten den Motiven der Kindermörderinnen nur wenig Beachtung geschenkt. Während der Aufklärungszeit trat dann das «Ehrenrettungsmotiv» stark in den Vordergrund, das auch Pestalozzi in seiner 1781 erschienenen Schrift «Über Gesetzgebung und Kindesmord» betonte. Als «Quellen» des Kindermordes führte er u.a. an: «Untreue und Betrug verführender Jünglinge» - «Die gesetzliche Strafe der Unzucht» - «Die Armuth» - «Die Furcht vor Eltern, Verwandten, Vormündern» - «Heuchlerischer Ehrbarkeitsschnitt» - «Innere und äußere Folgen früherer Laster» und «Die äußeren Umstände der Mädchen während ihrer Geburtsstunde » ${ }^{14}$, und er leitete aus diesen Erkenntnissen Forderungen an den Gesetzgeber und die Gemeinden ab. Diese Postulate sollen später erörtert werden (s. viertes Kapitel).

In vielen Teilen des Landes enthielten die Ordnungen besondere Strafandrohungen bei «Ehebruch, Nothzucht, Hurerey», aber auch für «uneheliche Beywohnung ». So bestimmte Art.26, Part.IV des Hamburger Stadtrechtes von 1603 (Von Strafe derer, die anderer Ehefrauen oder auch Jungfrauen wegführen) als Rechtsfolge die Todesstrafe durch Enthauptung:

«Der einem andern seine Ehe-Frau oder Tochter, freventlicher, vorsetzlicher oder betrieglicher Weise wider des Ehemannes oder der Eltern Willen heimlich oder öffentlich entführt, wie dann auch diejenigen, die einer Jungfrauen, Ehefrauen oder Witwen, wider der selbigen Willen mit Gewalt ihre Ehre nehmen, oder dieselbe nothzüchtigen, haben damit ihr Leben verwirkt, und wird denselben das Haupt abgeschlagen ",

während Ar. 28 (Strafe derjenigen, die Jungfrauen, Wittwen, oder Mägde verunehren, oder beschlafen) verlangte, daß diese Frau zu ehelichen oder nach ihrem Stande ein «Brautschatz zu entrichten sei»; auch war geboten, für ein Kind, das aus dieser Verbindung hervorgegangen war, «die Alimenta und Unterhalt zu schaffen ». Die Kurfürstliche Sächsische Landesordnung vom 1. September 1666 drohte Gefängnisstrafe an, wenn verlobte Personen «vor dem oeffentlichen Zusammen-geben und Trauen sich fleischlich mit einander einlassen ..., wenn gleich auch keine Schwaengerung daraus erfolget». ${ }^{15}$

In Hamburg gehörte der überwiegende Teil der ledigen Mütter der «ärmsten Volksklasse» an, wie es Rambach in seiner Schrift «Versuch einer physisch- 
medizinischen Beschreibung von Hamburg» erwähnt ${ }^{16}$. Es handelte sich hier hauptsächlich um Arbeiterinnen aus den Kattun-Fabriken. In diesen Fabriken wurden im Auftrage von Handelsfirmen Baumwollstoffe bedruckt. Bei den wertvolleren Kattunen mußten die Motive in den Stoff «eingeschildert» werden: sie wurden bemalt. Dazu stellten die Fabrikanten sogenannte «Schildermädchen», ungelernte Arbeiterinnen, ein. Sie hatten nur die Aufgabe, bestimmte Teile des Motivs mit einem Haarpinsel anzumalen. Im 18. Jahrhundert gab es in Hamburg und in den benachbarten Gemeinwesen Altona und Wandsbek je nach der Konjunkturlage 20-30 Fabriken mit bis zu 5000 Arbeitern und vor allem Arbeiterinnen. Im Winter mußte aus Witterungsgründen - fehlende Sonne, verdeckter Bleichrasen, zugefrorenes Wasser - die Arbeit fast ganz eingestellt werden. Unter den Beschäftigten herrschte dann bittere Not, wenn man in den Sommermonaten nicht in der Lage gewesen war, einiges vom Lohn zu sparen ${ }^{17}$.

Weiterhin handelte es sich bei den ledigen Müttern vielfach um Dienstmädchen. Auch hier war in den meisten Fällen die wirtschaftliche Notlage - denn die Dienstmädchen verloren in der Regel bei Eintritt einer Schwangerschaft ihre Stellung und mußten zudem mit Verweisung aus der Stadt rechnen, wenn sie ihrer Herrschaft von der Schwangerschaft Meldung erstatteten - das Motiv zur Tötung des Neugeborenen. Oft gelang es jedoch den Mädchen, die Schwangerschaft geheimzuhalten.

In Einzelfällen wurden auch Ehefrauen des Kindermordes überführt. Es handelte sich hier jedoch in der Mehrzahl um Kinder, die in «ehebrecherischen Verhältnissen» gezeugt worden waren. Nur vereinzelt wurden Fälle bekannt, wo das Neugeborene gemeinsam von den Eltern ausgesetzt bzw. getötet worden war. Auch hier waren wirtschaftliche Gründe oft die Ursache. Gleichfalls ist von Fällen von Aussetzungen von Totgeburten berichtet worden, in denen eine strafbare Handlung nicht vorlag. Die Aussetzung erfolgte hier lediglich, weil man die Begräbniskosten nicht zahlen konnte. So wurde z.B. im April 1787 ein totes, «schwer verstümmeltes Kind» auf dem Michaelis-Kirchhof gefunden. Das kleine Mädchen lag in einer "Candis-Lade», und jemand hatte die Schachtel «ohne Erlaubnis der Kirche und ungenügend begraben». Aus dem Sektionsprotokoll des Physikus ging hervor, daß «die Verstümmelung von dem Geburtshelfer mit seiner Zange oder ähnlichem Instrument» verursacht worden war. Der Physikus war jedoch nicht bereit zu entscheiden, ob das Kind in der Gebärmutter schon tot und nur mit Zwang - «mit Zangen» - herausgeholt oder ob die Wunde «von einer mörderischen Hand» bewirkt worden war. Weitere Untersuchungen deckten dann den wahren Umstand auf: Das Kind war zwar nicht ehelich gezeugt; die Eltern lebten «in wilder Ehe» schon seit mehreren Jahren zusammen 
und hatten bereits zwei totgeborene Kinder (!), die beide ordnungsgemäß bestattet worden waren. Der Geburtshelfer hatte nach Anforderung durch eine Hebamme das im Uterus schon tote Kind mit Zangen entbunden. Der Grund, warum das Kind nicht ordnungsgemäß begraben wurde, war einfach der, daß die Eltern zu arm waren, um ein passendes Begräbnis zu bezahlen. Deshalb hatte der Vater «sein Kind aus großer Noth in eine Candis-Lade gelegt und selbst auf dem großen Michaelis-Kirchhof hingesetzet ». ${ }^{18}$

\section{Maßnahmen zur Vorbeugung des Kindermordes}

Welche Maßnahmen wurden nun in dieser Zeit von der Obrigkeit ergriffen, um mit der Vermehrung dieser Delikte fertig zu werden? Für Hamburg können drei Maßnahmengruppen unterschieden werden, die zeitlich nacheinander versucht wurden, jedoch in Einzelbereichen Überschneidungen aufwiesen: karitative Maßnahmen und «moralische Kampagnen», sodann gesetzliche, schließlich gesundheitspolitische und sozialmedizinische Maßnahmen.

1597 wurde in Hamburg das Waisenhaus gegründet. Die Anstalt war bestimmt für verwaiste, unvermögende Bürgerkinder und Findlinge. Die Einnahmen bestanden aus Spenden, Sammlungen und Zinsen. Die Anstalt enthielt Platz für ungefähr 750 Kinder.

Schon im Jahre 1708 wurde von Jobst von Overbek ein «Torno», ein drehbarer Kasten, in dem Säuglinge dem Findelhaus anonym übergeben werden konnten, gestiftet, vorwiegend in bester Absicht, dem Kindermord - besonders unehelicher Kinder - vorzubeugen. In einem «Mandat bey Gelegenheit des beym Waysen-Hause aufgerichteten Torno» ist die rasche Zunahme des Kindermordes beschrieben; es wurde hier der Torno vorgestellt als ein Schutzmittel gegen Kindermord $^{19}$. C.Mönckeberg, Prediger in Hamburg, schrieb später über diese Initiative Overbeks, der auch in anderen Bereichen als großzügiger Spender sich hervorgetan hatte ${ }^{20}$ :

«Allein nicht nur für den Schmuck des Gotteshauses war Overbek bereit, sein Vermögen anzuwenden. Er hatte schon, als er Provisor am Waisenhause war, ein herzliches Mitleid gehabt mit den armen Kindern, die nicht Waisen waren, weil sie nicht durch den Tod ihre Eltern verloren hatten, sondern die selbst durch die Mütter, die kein mütterlich Herz hatten, dem Tode übergeben wurden. Eine Anstalt, wie sie bei dem Waisenhause in Venedig schon seit Jahrhunderten bestand, wie Rom sie besaß und Padua, ja, auch die deutsche Kaiserstadt Wien, wollte er gerne auch für seine Vaterstadt gründen. Einen Torno wollte er am Hause angebracht sehen, d. h. eine Drehmaschine, wodurch ein Kasten, der ein Kind fassen konnte, umgedreht werden konnte, so daß das Kind im Hause sich befand und durch den Klang einer Glocke angemeldet wurde, ohne daß der, welcher das Kind auf der Straße hineinlegte, bemerkt wurde. Auf diese Art konnten die 
Rabenmütter, wie er sie nannte, sich ihrer Kinder entledigen, und das häufige Kindermorden, wie das gefährliche Weglegen der neugeborenen Kinder wurde verhindert, ohne einen Mord zu begehen, und die Kinder wenigstens wurden gerettet».

Über das weitere Schicksal dieses Tornos heißt es dann:

«Overbek freute sich des Gelingens seines Werkes. Allein leider war schon durch die Verkündigung, die selbst auch von der Kanzel geschah, der neuen Einrichtung der Todesstoß gegeben. Eine große Anzahl Mütter glaubten nun berechtigt zu sein, sich ihrer Kinder zu entledigen. Man legte Kinder in den Torno, die schon zwei Jahre und darüber waren, preßte sie mit Gewalt durch den Torno; ja, auch von der Umgegend wurden Kinder in die Stadt gebracht, um hineingelegt zu werden. So vermehrte sich die Zahl der Kinder schon in den ersten Monaten auf eine solche Weise, daß die Provisoren des Waisenhauses in Angst geriethen, da sie nicht wußten, wie sie die Kinder ernähren sollten ».

Im Jahre 1727 wurde der Torno wieder aufgehoben, da trotz erneuter Mandate des Senates die Mißbräuche nicht abgestellt werden konnten.

Da es in der Hauptsache neben Arbeiterinnen auch Dienstmädchen waren, bei denen eine uneheliche Schwangerschaft eintrat, sprach man von der «Unmoralität und Entartung der Gesinde», und es wurden viele Pläne für die «moralische Verbesserung» des Gesindes vorgeschlagen. Die «Patriotische Gesellschaft» förderte diesen Gedanken, indem sie eine Preisschrift unter dem Titel ausschrieb: «Welches sind die hauptsächlichsten Quellen von dem Sitten-Verderben der Bediensteten beiderlei Geschlechts? Und wie kann demselben zu ihrem eignen sowohl, als der Herrschaften Besten, ohne gesetzlichen Zwang gewehrt werden?» - Es wurden 13 Preisschriften eingereicht. Eines der am häufigsten empfohlenen «Heilmittel» gegen diese Sittenverderbnis war die Betonung einer «strengeren, zweckmäßigen, geistlichen Bildung» für Dienstboten durch spezielle Gesindepredigten. Weiterhin empfahl man die Schaffung einer «Gesinde-Ordnung» im Zusammenhang mit der Forderung nach einer «Vereinigung vieler Herrschaften untereinander zu einem gemeinschaftlichen Zweck der Besserung ihrer Untergebenen in Form einer Patriotischen Gesellschaft zur Sittenverbesserung des Dienstvolkes». Weiterhin sollte ein «Gesinde-Comptoir» gegründet werden, in dem Berichtblätter über die einzelnen Bediensteten geführt wurden mit den entsprechenden Eintragungen. Es sollte also hier eine Kontrolle ausgeübt werden. Außerdem wurde gefordert, «einheimisches Gesinde dem ausländischen vorzuziehen». Die fremden Mägde wurden als Gefahr der schlechten Beeinflussung des einheimischen Dienstpersonals angesehen, weil «die schon allenthalben in der Welt herumgeschwärmt haben und hier zuletzt ihre Zuflucht suchen und finden ». ${ }^{21}$

Die zweite Maßnahme war dann die strafrechtliche, wie schon im dritten Kapitel ausgeführt wurde. Insbesondere sind hier die Mandate zu erwähnen, die die Meldepflicht und Strafandrohung für verheimlichte Schwangerschaft sowohl 
für Schwangere selbst wie auch für die Mitwisser enthielten. Das Mandat vom 18. Oktober 1724, wurde 1765 erneuert $^{22}$; die wiederholten «Renovierungen» zeigen, daß die Erlasse auf die Dauer nicht wirksam waren.

Zum Teil in Ableitung aus diesen Mandaten, aber auch in der Erkenntnis, daß mit medizinischen Maßnahmen dieses Problem vielleicht eher bewältigt werden könnte, erhoffte man sich von einer Neuordnung des Hebammenwesens eine Verbesserung. Bereits der Reformator Johannes Bugenhagen hatte 1529 auf die Bedeutung der Bademütter hingewiesen und in der von ihm verfaßten Ordnung bestimmt:

«Diese können aber auch Kirchendienerinnen heißen, denn an ihrem Amte ist viel gelegen, daß es als erstes kundig und gewissenhaft, dann auch christlich dabei zugehe, gemäß der genauen Anweisung in der Braunschweiger Ordeninge. Danach soll ein Ehrbarer Rat ja stets so viele kundige und tüchtige und christliche Frauen zur Hand haben, wie hier für alle notwendig sind, denn fahrlässiges Versäumnis in diesen Dingen wäre sehr gefährlich. ${ }^{23}$

Die älteste spezielle Bademütter-Ordnung in Hamburg datiert aus dem Jahre 1654 und wurde 1661 neu gefaßt. Die nächsten zwei Ordnungen von 1704 und 1718 (Abb. 4) unterscheiden sich davon nur wenig. Die letztere hat dann - gelegentlich ergänzt und in einzelnen Maßnahmen verschärft - bis zum Erscheinen der «Instruction für die Hebammen der Stadt Hamburg und deren Gebietes » im Jahre 1832 Gültigkeit gehabt. Die weitreichende und längst überfällige Revision der städtischen Medizinal-Ordnung von 1818 hatte trotz ihres Umfangs keine neue Bademütter-Ordnung geschaffen. Diese wurde erst durch die vom Gesundheitsrat erlassene Vorschrift von 1832 verwirklicht ${ }^{24}$. Im $§ 112$ der MedizinalOrdnung waren insbesondere «die Verhinderung des Kindermordes und die Verbreitung venerischer Übel» als Gründe für eine nötige Neuordnung hervorgehoben worden.

Schon in der ersten Bademütter-Ordnung von 1654 gab es eine Pflicht für die Hebammen (Bademütter, Wehmütter), «alle Huren- und Spielkinder», d.h. alle von den Hebammen entbundenen außerehelichen Kinder, zu melden. Eine besondere Strafe von «100 Mark Lübisch und die Enthebung aus ihrem Amte» wurde angedroht, wenn sie dieser Meldepflicht nicht genügten. Auch im Bademütter-Eid von 1661 mußten sie schwören, «daß ich solches [Huren- und Spielkind] dem ältesten Herrn Gerichtsverwalter will ankündigen » ${ }^{25}$.

Weiterhin wurde unterstellt, daß es für die Bademütter am leichtesten wäre, den Namen des Schwängerers von der unverheirateten Frau zu erfragen, und sie wurden also dazu verpflichtet, «die schwangere Weibsperson mit höchstem Fleiß und Ernst zu befragen, den Vater des Kindes zu nennen, damit also der 

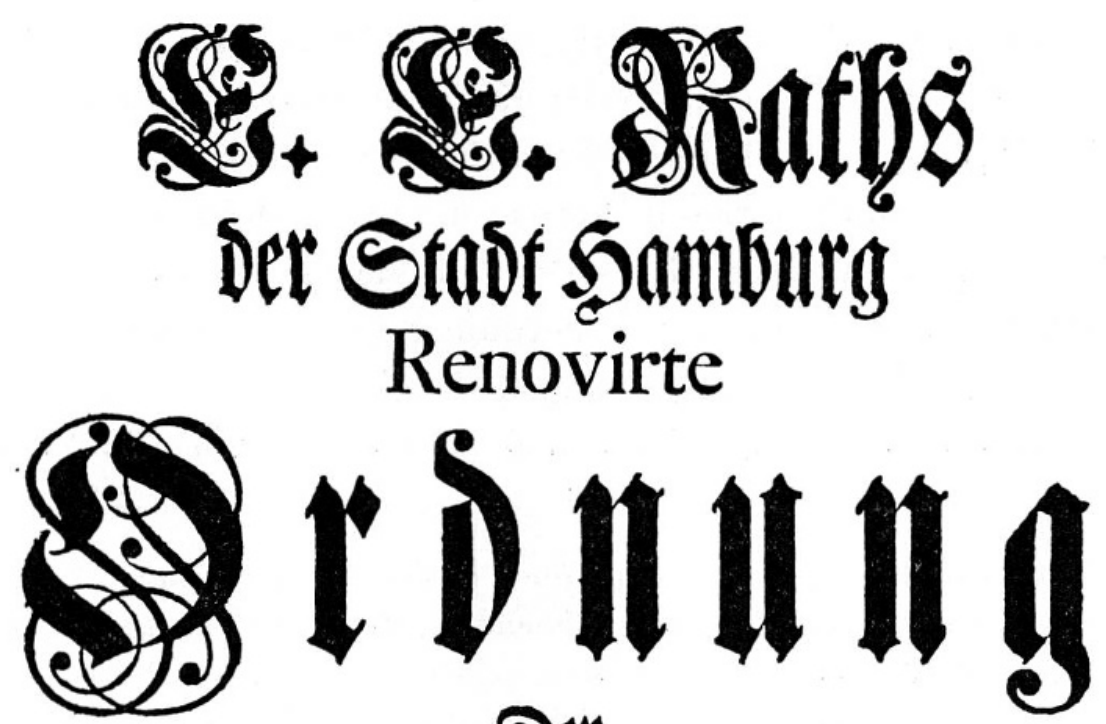

Det
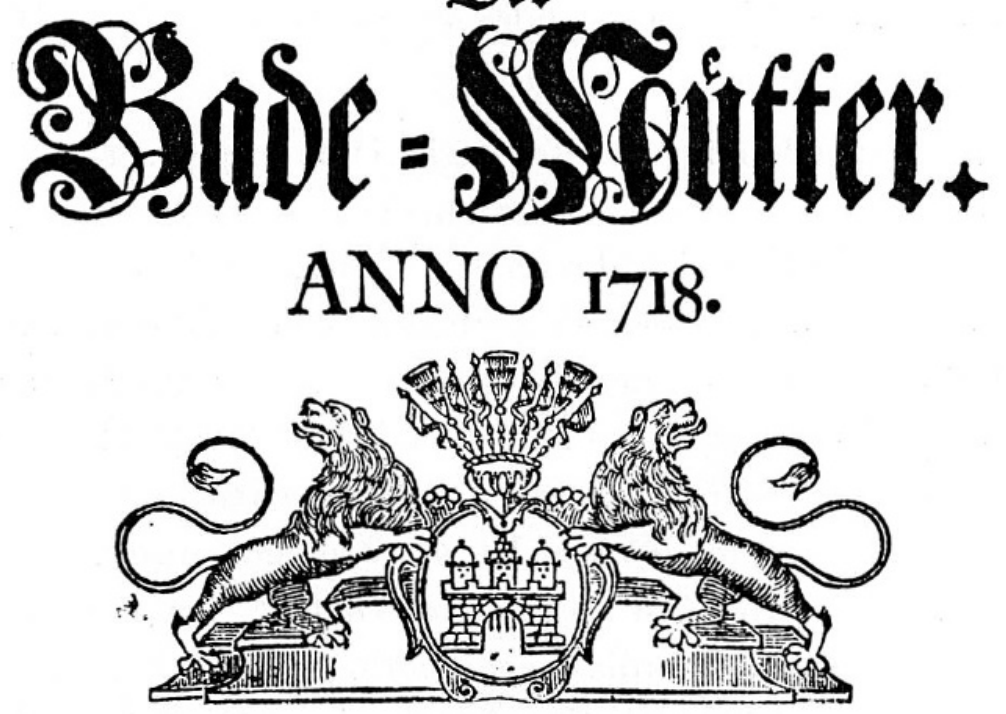

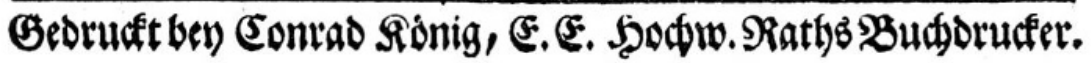

$A b b$. 4. Staatsarchiv Hamburg: Senat, Cl. VII Lit. $L^{\mathbf{b}}$ Nr. 3 Vol. 1

echte Vater namkündig gemacht und nicht verschwiegen werde ... und dasselbige dem ältesten Gerichtsverwalter mit getreuem Fleiß anzuzeigen und zu vermeldigen ${ }^{26}$

Die Verfügung, alle unehelichen Geburten anzumelden, galt für Hebammen und Geburtshelfer. An diese Verpflichtung wurde immer wieder erinnert, oft verstärkt nach erneuter Aufdeckung eines Kindermordes oder nach Auffindung eines ausgesetzten Kindes. Diese eindringlichen Hinweise erließ der Senat in den Zeitungen. Nachdrücklich wies er die Wehmütter darauf hin, daß sie gemäß Art.13 der Bademütter-Ordnung (1718) (Abb. 5) alle unehelichen Geburten, bei 


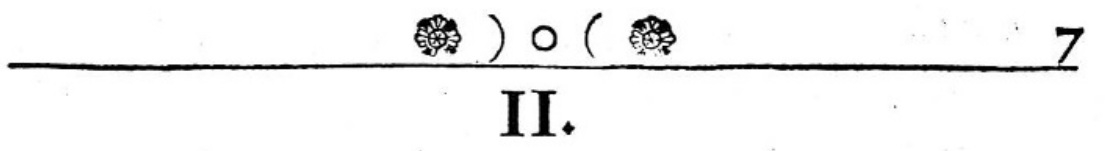

Damit wegen ber $\mathfrak{B e d i e n u n g ~ b e r ~ b e r ~ R i n o ~ : ~ T a u f f e ~}$

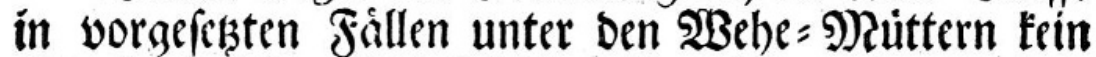
Streit entitibe; fo roll foldes allemabl von ber vorbers

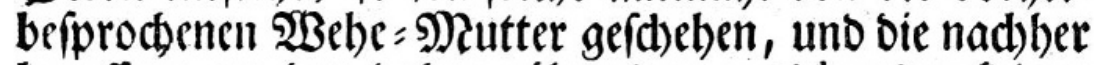
beruffene an Den Daber rubsenden accidentien feinen 2intbeil baben, fondern fich an Dem, was ibnen, Der bey oer (siebulbrt geleifteten affiftenks balber, gegeben worben, bignuigen laffen.

\section{I2.}

Eoll niemano ber $\mathfrak{B a d e}=$ Mutter fid unterfteben, eine anorre neue $\mathfrak{B a d e}=$ ) Nutter z Denn, Dấ fie filbft etwann fechszel)n Jabre Damit um= gegangell fen, bey Strafe Ein Sundert Mard Lubift).

\section{I3.}

Es follen aud fonft bie $\mathfrak{B a b e}=$ Muitter ifbem gelei: feten (Ev) in allen Puncten nachfommen, uno, Dem fel: ben zur folge, die SHur=und Epiel = Rinder Dem álteften Serrn (Gerid)to $=$ Berwalter felbften unfáumig anmel= Den, und da eine betreten wurbe, Dấ fie ibrem Eibe zu= gegen gebandelt bátte, bicielbe foll nicht allein in Ein Sundert Mard Lubiíd) Strafe bem (Seridhte verfallen fern, fondern auct) Daju ibres $\mathfrak{B a b e}=$ Mutter $=$ Amts fid) Daburd) verluftig gemactet baben.

\section{MBeil}

$A b b$. 5. Aus der Bade-Mütter-Ordnung von 1718

(Staatsarchiv Hamburg)

denen sie Hilfe geleistet hatten, ohne Ausnahme dem ältesten Gerichtsverwalter zu melden verpflichtet seien ${ }^{27}$.

Später wurde durch eine Verordnung vom 13. Februar 1788 diese Meldepflicht für Accoucheurs ${ }^{28}$ erweitert, und auch sie wurden wiederholt ermahnt, alle Geburten, nicht nur die ehelichen, bei denen sie Beistand geleistet hatten, «wöchentlich dem ältesten Herrn Prätori» anzuzeigen. Diese Ausdehnung der Meldepflicht für Accoucheurs erscheint besonders erwähnenswert, da ebenfalls eine Häufigkeit von Kindestötung infolge der Ungeschicklichkeit der Geburtshelfer festzustellen war. 
Besondere Meldepflichten bestanden für «Lehrlings-Wehmütter», d.h. für diejenigen Hebammen, die sich noch in der von der Bademütter-Ordnung festgesetzten vierjährigen Lehrzeit befanden. Sie wurden verpflichtet, alle Geburten, bei denen sie Beistand geleistet hatten, einer höheren Behörde, der Rats- oder Bademutter oder den Physici zu melden. Diese Meldepflicht erscheint neben dem Versuch, die Schulung der Lehrlings-Hebammen zu regulieren und zu überwachen, auch als geeignetes Mittel, die unehelichen Kinder ausfindig zu machen und hier in bezug auf die Kindestötung vorbeugend eingreifen zu können.

In diesem Zusammenhang ist noch die Tätigkeit der vereidigten Wehmütter bei der Taufe bemerkenswert. Für die Bestätigung der Ehelichkeit des Kindes, das die Hebamme entbunden hatte, war das «Zur-Taufe-Tragen» des Kindes ein Ehrenamt und eine Tätigkeit, wofür sie noch ein Trinkgeld von der Familie zusätzlich zu ihrem Entbindungshonorar erhielt. Dieses «Tragen zur Taufe» war nicht nur ein Brauch, sondern sollte auch äußerlich als Beweis dienen, daß «das Kind in der Ehe gezeugt worden war». Es war unbeeidigten Wehmüttern ausdrücklich untersagt, ein Kind zur Taufe zu tragen. Man wollte damit verhindern, daß uneheliche Kinder als eheliche deklariert wurden ${ }^{29}$.

Eine Besonderheit soll noch erwähnt werden, und zwar die Forderung für alle vereidigten Wehmütter: «Sie solle lesen und, wenn möglich, am besten auch schreiben können ». Die erste Erwähnung dieser Notwendigkeit findet sich schon in der Bademütter-Ordnung von 1654, wo vermerkt wurde, daß diejenige, die Bademutter werden wolle, sich bei den Physicis und der Ratswehmutter zu melden habe und «von ihnen in ein Buch eingeschrieben, dafern sie aber aufs wenigste nicht lesen kann, abgewiesen werde ». ${ }^{30}$

Es wurde sicherlich nicht erwartet, daß diese Frauen medizinische Fachbücher lesen würden, aber man verlangte von ihnen, daß sie i mstande waren, ein Exemplar der Bademütter-Ordnung zu lesen und auswendig zu lernen. Die Bedeutung dieser Voraussetzung wird klarer, wenn man die Wichtigkeit der Meldepflicht bedenkt.

Die neue Hebammen-Ordnung von 1832, als «Instruction für die Hebammen der Stadt Hamburg und deren Gebietes auf Befehl eines Hochedlen Raths in Gemäß̣heit $\S 112$ der Medizinal-Ordnung publiziert» ${ }^{32}$, enthielt auch die Übernahme neuer besonderer Pflichten für die Hebammen ${ }^{31}$. Die Instruktion umfaßte in sieben Abschnitten «Allgemeine Vorschriften über das moralische Verhalten der Hebammen» ( $\S 1-5)$, «Allgemeine Vorschriften für die Ausübung der Hebammenkunst» (§§6-15), «Besondere Vorschriften bei regelmäßigen Geburten» (§§16-35), «Besondere Vorschriften bei schweren und regelwidrigen Geburten» (§§ 36-64), «Besondere Vorschriften bei regelwidrigen und krankhaften Zufällen 
nach der Entbindung und dem Wochenbett» (§§ 65-81), «Besondere Vorschriften bei regelwidrigen und krankhaften Zufällen oder angeborenen Fehlern des Kindes» (§§ 82-94) sowie den Eid der Hebammen. Diese Ordnung zeichnet sich besonders durch einen ausführlichen medizinischen Teil aus, während sich ihre Vorläufer in Anweisungen über den Lebenswandel der Hebammen, das Verhalten bei Nottaufen usw. erschöpft hatten und medizinische Instruktionen weitgehend vernachlässigten.

\section{Zusammenfassung}

Die im 18. Jahrhundert allgemein festgestellte Zunahme der Delikte von Kindesaussetzungen und Kindermord ist auch in der Hansestadt Hamburg zu beobachten. In den leider nur noch lückenhaft vorhandenen Archivalien konnten für einige Zeiträume des 18. Jahrhunderts anhand von Aufzeichnungen über die Zahl der tot gefundenen Kinder und anhand von Senatsprotokollen über Verurteilungen überführter Täter diese Delikte nachgewiesen werden. Eine eigentliche Kriminalstatistik im heutigen Sinne war auch in Hamburg nicht vorhanden, und die lückenhaften tabellarischen Angaben geben nur ein ungefähres Bild der Kriminalität auf diesem Gebiet, da gerade bei diesem Delikt eine hohe Dunkelziffer anzusetzen ist.

Eine besondere Rolle spielt in diesem Zusammenhang das Delikt der «verheimlichten Schwangerschaft», das, wie vielerorts, auch hier als Vorstadium zu einer geplanten Kindestötung angesehen wurde. Daher war es auch erforderlich, in Anlehnung an die entsprechende Gesetzgebung für Kindermord in einem Mandat dieses Delikt besonders herauszuarbeiten und eine Strafandrohung sowohl für die Schwangere selbst als auch für die Mitwisser auszusprechen. Die Gesetzgebung selbst mußte in Hamburg in Anlehnung an die «Carolina» (Peinliche Halsgerichtsordnung - P.H.G.O.) erfolgen, da das Lübische und damit das Hamburgische, von Lübeck beeinflußte Recht keine spezifischen Strafmaßnahmen für Kindermörderinnen vorsah. Die entsprechenden Artikel des Hamburger Stadtrechtes von 1603 übernahmen dann die $\S \S 135$ und 137 der P.H.G.O.

Die Gesetzgebung der «Carolina» forderte für die überführte Kindermörderin die Todesstrafe und setzte damit das Strafmaß für dieses Delikt dem Brudermord gleich. Auch in Hamburg wurde durch diesen Artikel die Todesstrafe angedroht; bei Auswertung des vorhandenen Archivmaterials gewinnt man jedoch den Eindruck, daß zwar die Todesstrafe hie und da verhängt wurde, in der Mehrzahl der Fälle jedoch eine Abwandlung in Zuchthaus- oder Gefängnisstrafe vorgenommen wurde. Hier spielten das zu Anfang des 17.Jahrhunderts errichtete 
Zucht-, Werk- und Armenhaus sowie das Spinnhaus für die Unterbringung der Verurteilten eine besondere Rolle.

Die Strafandrohung war vielerorts eine der Hauptmaßnahmen zur Eindämmung dieser Straftaten. Für Hamburg ergab sich, daß zwar die strafrechtlichen Maßnahmen ebenfalls eine Rolle spielten; vielleicht sogar noch bedeutungsvoller aber waren karitative und insbesondere medizinalgesetzgeberische und sozialmedizinische Bestrebungen. Eine strafrechtliche Vorbeugungsmaßnahme in einer reichhaltigen Unzuchtsgesetzgebung, die bewirken sollte, daß es infolge der angedrohten Strafe gar nicht erst zu den verbotenen außerehelichen Geschlechtsbeziehungen kam, wurde in Hamburg nur bedingt durchgeführt. Strenger gehandhabt wurde die Gesetzgebung hinsichtlich der Verheimlichung der Schwangerschaft unverehelichter Weibspersonen, wie sie sich z. B. auch in einer NassauWeilburgischen Verordnung vom 11. März 1750 oder in einem Edikt vom 30. August 1720 der preußischen Gesetzgebung niederschlug ${ }^{33}$. Auch in Frankreich konnte das Unterlassen der Meldepflicht schwere Folgen haben. Dort wurde nach einem Gesetz von Heinrich II., schon aus dem Jahre 1556, die Todesstrafe für die Frau gefordert, der dieses Delikt nachgewiesen werden konnte ${ }^{34}$.

In Hamburg versuchte man durch immer wieder vorgenommene Bekanntmachungen der seit 1654 geltenden Hebammen-Ordnung, die «heimliche Schwangerschaft» offenkundig zu machen und damit schon einem Kindermord vorzubeugen. Die Regelung, daß alle unehelichen Geburten angemeldet werden mußten, galt für Geburtshelfer wie für Hebammen. Sie wurde häufig, oft bei Gelegenheit eines entdeckten Kindermordes oder nach der Auffindung eines «weggelegten Kindes», öffentlich in Erinnerung gerufen. Der Senat versprach sich hiervon am nachhaltigsten einen Rückgang dieser Delikte. Er verfolgte dabei auch die Absicht, die Anzahl der unehelichen Kinder auf ein Minimum zu reduzieren, da diese ja der Stadt zur Last fielen.

\section{Anmerkungen (Quellen und Literatur)}

1 Wächtershäuser, W., Das Verbrechen des Kindesmordes im Zeitalter der Aufklärung. Eine rechtsgeschichtliche Untersuchung der dogmatischen, prozessualen und rechtssoziologischen Aspekte. In: Kaufmann, E., und H. Holzhauer, Quellen und Forschungen zur Strafrechtsgeschichte, Bd. 3, Erich Schmidt-Verlag, Berlin 1973, S. 109.

${ }^{2}$ Senat (auch Rat genannt) war zu jener Zeit Regierung, oberstes Gericht und Verwaltungsspitze der reichsunmittelbaren Stadt Hamburg.

${ }^{3}$ Prätor (Behörde: Prätur) war im jährlichen Wechsel ein Ratsherr mit polizeilichen und gerichtlichen Funktionen (Gerichtsverwalter).

4 Blank, J. F., Sammlung der von E. Hochedlen Rathe der Stadt Hamburg so wol zur Handhabung der Gesetze und Verfassungen als bey besonderen Eräugnissen in Bürger- und 
Kirchlichen, auch Cammer-, Handlungs- und übrigen Polizey-Angelegenheiten und Geschäften vom Anfange des siebenzehntenJahr-Hunderts bis auf die itzige Zeit ausgegangenen allgemeinen Mandate, bestimmten Befehle und Bescheide, auch beliebten Aufträge und verkündigten Anordnungen, J. C. Piscator, 3.Teil, Hamburg 1764, S. 1673.

5 Staatsarchiv Hamburg: Senat, Cl. VIII Nr. X, Jgg. 1742-1800.

${ }^{6}$ Zahlenangaben für die Jahre 1712-1715 aus: Hamburgisches Diarium; für die Jahre 1768 bis 1800 aus: Hamburgische Addreß-Comptoir-Nachrichten. Wegen der Einwohnerzahlen siehe Heinrich Reincke, Hamburgs Bevölkerung. In: Forschungen und Skizzen zur Hamburgischen Geschichte, Hamburg 1951, S. 167 ff.; vgl. auch Statistisches Jahrbuch 1953/54, Freie und Hansestadt Hamburg, S. 6.

7 Fleete waren zahlreiche schiffbare, schmale Kanäle, die das Hamburger Stadtgebiet durchzogen; sie dienten der Wasserversorgung und zu Transportzwecken. Sie standen mit den Flüssen Alster und Elbe in Verbindung.

8 Hufton, O., The Poor in Eighteenth-Century France, 1750-1789, Oxford 1974, S. 321.

9 Aus: Handwörterbuch zur deutschen Rechtsgeschichte, Stichwort «Kindestötung» (Wächtershäuser), Sp. 736-74.1.

10 Staatsarchiv Hamburg: Senat, Cl. VII Lit. $\mathrm{M}^{\mathrm{e}}$ Nr. 8 Vol. $1^{\mathrm{b}}$. Mandat gegen Kindermord.

11 Klefeker,J., Sammlung der Hamburgischen Gesetze und Verfassungen in Bürger- und Kirchlichen, auch Cammer-, Handlungs- und übrigen Polizey-Angelegenheiten und Geschäften samt historischen Einleitungen, 5.Teil, Hamburg 1768, S. 500.

${ }^{12}$ Staatsarchiv Hamburg: Senat, Cl.VII Lit. $M^{\mathrm{a}}$ Nr. 5 Vol. $3^{\mathrm{d}} 6$. Senatsprotokoll vom 26. März 1770.

13 Kerner, G., Über das Hamburgische Entbindungshaus und das Entbindungswesen der Armen-Anstalt, Hamburg 1810.

14 Wächtershäuser, W., a. a. O., S. 54 ff.

15 Wächtershäuser, W., a.a. O., S. $155 \mathrm{ff}$.

16 Rambach,J.J., Versuch einer physisch-medizinischen Beschreibung von Hamburg, Carl Ernst Bohn, Hamburg 1801, S. 263.

17 Heß,J. L., Hamburg topographisch, politisch, historisch beschrieben, 2. Teil, Hamburg 1789, S. 245-247.

18 Staatsarchiv Hamburg: Senat, Cl. VII Lit. L ${ }^{\mathrm{b}}$ Nr. 3 Vol. 5.

19 Blank, J. F., Sammlung, 2.Teil, 1763, S. 631-634.

${ }^{20}$ Mönckeberg, C., Jobst von Overbek. In: Zeitschrift des Vereines für hamburgische Geschichte, 4. Bd., August Meißner, Hamburg 1858, S. 321 ff.

21 Verhandlungen und Schriften der Hamburgischen Gesellschaft zur Beförderung der Künste und nützlichen Gewerbe, 2. Bd., Hamburg 1793, S. 61-176.

22 Blank, J. F., Sammlung, 6. Teil, 1774, S. 90.

${ }^{23}$ Bugenhagen, J., Der Ehrbaren Stadt Hamburg Christliche Ordnung 1529, Hamburg 1976, S.123 (Arbeiten zur Kirchengeschichte Hamburgs, Bd.13). Herausgegeben und übersetzt von Hans Wenn.

${ }^{24}$ Medizinal-Ordnung für die freie Hansestadt Hamburg und deren Gebiet vom 20. Februar 1818. In: Hamburgische Verordnungen 1818, S.44. Instruction für die Hebammen der Stadt Hamburg und deren Gebietes vom 30. März 1832. In: Hamburgische Verordnungen 1832, S. 52.

${ }^{25}$ Staatsarchiv Hamburg: Senat, Cl. VII Lit. L ${ }^{\text {b }}$ Nr. 3 Vol.1. 
${ }^{26}$ Staatsarchiv Hamburg: Senat, Cl. VII Lit. L ${ }^{b}$ Nr. 3 Vol. 1. Eid der Bademütter von 1661.

${ }^{27}$ Staatsarchiv Hamburg: Senat, Cl. VIII Nr. X. Protokoll vom 7. Mai 1787.

${ }^{28}$ Accoucheurs $=$ Geburtshelfer.

${ }^{29}$ Blank,J. F., Sammlung, 2.Teil, 1764, S. 910. Renovierte Bademütter-Ordnung vom 9. November 1718.

${ }^{30}$ Staatsarchiv Hamburg: Senat, Cl. VII Lit. L ${ }^{\text {b }}$ Nr. 3 Vol.1, Art. 5 : Bademütter-Ordnung von 1654.

${ }^{31}$ Staatsarchiv Hamburg: Senat, Cl. VII Lit. $\mathrm{L}^{\mathrm{b}}$ Nr. 23 a Vol. 27.

${ }^{32}$ Staatsarchiv Hamburg: Senat, Cl. VII Lit. L ${ }^{b}$ Nr. 23 a Vol. 27.

33 Wächtershäuser, W., a. a.O., S.139.

${ }^{34}$ Hufton, O., a.a. O., S. 320.

\section{Summary}

This article hopes to illustrate the relationship between the crimes of infanticide and child abandonment and the less well-known crime of concealing an illegitimate pregnancy. Although criminal statistics in a modern sense certainly do not exist for 18th century Hamburg, it is possible by using other available archival material, such as the protocols of the city's major ruling council, to develop some picture of not only the crime but also of the major criminal involved - the unwed mother. This article also deals with the measures taken to prevent these crimes. Although the discussion of punitive measures cannot be neglected, here a special attempt has been made to discuss the philanthropic, educational and medical means utilized to nip crime in the bud. Particular attention is given to the role of medical personnel, the city's mid-wives and "Geburtshelfer" (male mid-wives), in "uncovering" illegitimate pregnancies and registering them with the proper authorities. It is here asserted that such methods were not only directed at preventing infanticides but also at trying to minimize the number of illegitimate and homeless children, who inevitably burdened the city's charitable institutions.

Priv.-Doz. Dr. med. Heinrich Rodegra

Institut für Geschichte der Medizin

D-2000 Hamburg 20

Martinistraße 52

Mary Lindeman, M.A.

Department of History

University of Cincinnati

Cincinnati/Ohio 45221

Dr. jur. Martin Ewald

Staatsarchiv Hamburg

D-2000 Hamburg 36

ABC-Straße 19A 